\title{
STABILITY OF SWITCHED LINEAR SYSTEMS WITH POISSON SWITCHING
}

\author{
B. HANLON*, V. TYURYAEV ${ }^{\dagger}$, AND C. MARTIN ${ }^{\ddagger}$
}

\begin{abstract}
We examine the stability of continuous time linear switched systems when the switching times are governed by a Poisson process. We construct an infinite family of polynomial Lyapunov functions that governs the stability of the expected value, as well as higher order moments. The analysis is accomplished by converting the problem to a stochastic process and analyzing this process.
\end{abstract}

Key words: random products, stochastic systems, switching systems, stability

1. Introduction. The study of multimode systems capable of fast switching rates is indeed very challenging. Stochastic control provides a framework for modeling and analysis if some probabilistic data on switching patterns are available. In this paper, we take a different path and ask the simple question: If switching between modes is governed by a Poisson process, how can we carry out a stability analysis of the system? Basic tools to carry out such an analysis are a prerequisite to developing a successful theory of stabilization of switching systems whose switching times are governed by a known probability distribution. From a heuristic viewpoint, stability is a necessary property to accompany solutions to all control paradigms, and, in this sense, we are taking a step to develop new control paradigms. It is our hope that in the future we will be able to answer questions such as stabilization, trajectory planning with closed-loop stability, and tracking.

The human muscular control system provides a canonical example of a system with rapid switching. Most of the muscles of the body occur in opposing pairs and only one muscle of the pair can be actively used at any one instant. In a simple movement, such as the movement of the eye in a horizontal plane, the exterior rectus muscle controls the temporal motion and the interior rectus controls the nasal motion. To track an object that is oscillating, using only eye movements, the control switches between the internal and external rectus muscle. See [25] for a more complete analysis of eye movement. In more complicated motion there may be many muscle pairs involved that are switching between opposing muscles and even opposing muscle groups. One only needs to watch a gymnast performing on the pommel horse to see rapid switching between muscle groups. It is reasonably clear in the pommel horse

\footnotetext{
${ }^{*}$ Department of Statistics, University of Wisconsin, Madison, WI 53706-1510, E-mail: hanlon@stati.wisc.edu

${ }^{\dagger}$ Department of Mathematics and Statistics, Texas Tech University, Lubbock, TX, 79409-1024, USA, E-mail: vadim.Tyuryaev@ttu.edu

${ }^{\ddagger}$ Department of Mathematics and Statistics, Texas Tech University, Lubbock, TX, 79409-1024, USA, E-mail: clyde.f.martin@ttu.edu, the author wishes to acknowledge the hospitality of Kyoto University during the time this research was done.
} 
that lack of coordination during switching will result in an instability. Significant work remains in the analysis of the stability of even the simplest aspects of the human muscular control system; we expect to find that the role of the switching pattern is very important.

Switched and multimode systems have received a fair amount of attention from the control theory community. However, much of the work has focussed on finding an appropriate switching strategy in order to stabilize the system; see, for example, [18], [24], and [28]. More generally, variable structure control is a well-developed area of research and its aim is essentially to find a stabilizing switching strategy. On the other hand, the recent work of Branicky [2] is closely related to our work. He assumes that a class of switching paths have been enumerated and asks, When is the system stable for all switching paths? He has shown that if the system admits a variant of a time-varying Lyapunov function, then it is asymptotically stable. In [5] the goal was to determine when the system is stable for all possible switching patterns and to construct a Lyapunov function in the case that it was stable. Our work aims at addressing the complimentary problem of stability when the class of switching system is assume to satisfy a probabilistic law.

Another class of problems closely related to ours is that of stabilizing a family of linear systems in such a way that the closed-loop systems admit a common quadratic Lyapunov function; see, for example, [1], [11], [22], [12]. The essence of this set of work is captured in [1] where it was shown that for the family

$$
\dot{x}=A_{\gamma} x+B_{\lambda} u, \quad x \in R^{n}, \quad \gamma \in \Gamma, \quad \lambda \in \Lambda
$$

one can reduce the problem to that of finding a common quadratic Lyapunov function for a family of uncontrolled systems on a certain subspace of $R^{n}$. Interestingly, an example in [5] illustrates a limitation of the stabilization problem of Barmish [1], namely that asking for a quadratic Lyapunov function may be too restrictive. In general, one needs to ask for a Lyapunov function which may be of degree higher than two. In this paper we establish that even if we allow arbitrary polynomial Lyapunov functions this still may not suffice. In the early development of the geometric theory of nonlinear control systems, it was routine to consider control inputs to be piecewise constant functions. Therefore, if one were to study controllability of a smooth nonlinear control system $\dot{x}=f(x, u)$, it is natural to consider the collection of vector fields $D=\{f(x, u): u \in U\}$, where $U$ is some admissible control set, and then study the end points of all curves obtained by concatenating integral curves of vector fields in $D$. Therefore, it can be thought of as a system with a multitude of modes, each corresponding to a vector field in $D$.

A recent survey by Lin and Antsaklis, [15], gives a rather complete overview of the field. The main theorem cited there on stability for discrete time switched linear systems, (their Theorem 6, [14]), requires that $2^{n}$ matrix norms must be calculated. 
In this paper we give a simpler calculation but one that will only hold for "almost all" switching sequences. An excellent and readable source for the general theory of switched linear systems is the monograph by Liberzon, [13]. In his book the primary focus is on continuous dynamics but the concepts are similar for discrete time. However, for discrete time switched systems the literature is much older than the systems theory literature, going back to at least the early 1960's with the publication of the papers of Furstenberg and Kesten, [8, 9], in the area of products of random matrices, or, if you like, the random products of matrices. In the statistical literature on random products "almost all" is the standard condition.

The motivation for studying this problem came from reading a series of papers by S.A. Murphy, [19, 20, 21], on dynamic treatment regimes and associated dynamic clinical trials. In fact, the theses by Du and Wang, [6, 26], were directly related to this topic. The central idea behind dynamic treatments is that the treatments are switched between two or more treatments types in hopes of producing an improvement over any single treatment. A natural model for such a course of treatments lies in the area of switching systems.

In this paper we consider the switched linear system

$$
\dot{x}=(\delta(t) A+(1-\delta(t)) B) x
$$

where

$$
\delta(t) \in\{0,1\}
$$

for all $t$. This system was studied by Dayawansa and Martin, [5], and they proved that the system is stable for all switching sequences $\delta(t)$ if and only if the two systems

$$
\dot{x}=A x \quad \text { and } \quad \dot{x}=B x
$$

have a common Lyapunov function. They also show that for this system there exists a pair of matrices $(A, B)$ for which the switching system is stable but that there is not a common quadratic Lyapunov function. This severely limits the usefulness of that result.

In this paper we analyze the problem from the viewpoint of stochastic systems and obtain results regarding a modified version of this problem. At the same time we obtain results about the random products of matrix exponentials. Our primary goal is to obtain results for pairs of matrices, but the results can be restated for any finite number of matrices by considering the system

$$
\dot{x}=\left(\delta_{1}(t) A_{1}+\cdots+\delta_{k}(t) A_{k}\right) x,
$$

where for each fixed $t, \delta(t)$ is a random vector in $\mathbb{R}^{k}$ which satisfies,

$$
\begin{aligned}
\sum_{i=1}^{k} \delta_{i}(t) & =1 \\
\delta_{i}(t) & \in\{0,1\} .
\end{aligned}
$$


In other words, for each fixed $t, \delta(t)$ is a random element chosen from the canonical basis vectors of $\mathbb{R}^{k}$.

In fact, throughout this paper we focus on switching sequences generated by a Poisson process. The resulting system is given by

$$
\begin{aligned}
& d x=((z(t) A+(1-z(t)) B) d x \\
& d z=(1-2 z(t)) d N
\end{aligned}
$$

where $N$ is a Poisson process of rate $\lambda$. Note that the process $d z$ takes on only the value of 0 or 1. This work is based on the notes of Roger Brockett, [4]. The goal of the first part of the paper is to obtain differential equations for all of the moments of $d x$. This will be done using a device that Brockett has used in the study of Volterra systems, [3].

2. Calculating Expected Value. We rewrite the system (1.5) as

$$
d\left(\begin{array}{l}
x \\
z
\end{array}\right)=\left(\begin{array}{c}
(z A+(1-z) B) x \\
0
\end{array}\right) d t+\left(\begin{array}{c}
0 \\
1-2 z
\end{array}\right) d N .
$$

Taking expectations we then have

$$
\frac{d}{d t}\left(\begin{array}{c}
E(x)(t) \\
E(z)(t)
\end{array}\right)=\left(\begin{array}{c}
(A-B) E(z x)(t)+B E(x)(t) \\
(1-2 E(z)(t)) \lambda
\end{array}\right)
$$

To evaluate this we must have an equation for the expected value of $z x$. To obtain this we use the Itô rule and calculate the stochastic equation for $d(z x)$. Recall that for a function $\psi(x)$ the Itô rule is

$$
d \psi(\hat{x})=\left\langle\frac{\partial \psi}{\partial \hat{x}}, f(\hat{x})\right\rangle d t+[\psi(\hat{x}+g(\hat{x}))-\psi(\hat{x})] d N .
$$

Here we have

$$
\hat{x}=\left(\begin{array}{l}
x \\
z
\end{array}\right), \quad f(\hat{x})=\left(\begin{array}{c}
(z A+(1-z) B) x \\
0
\end{array}\right) g(\hat{x})=\left(\begin{array}{c}
0 \\
1-2 z
\end{array}\right)
$$

and

$$
\psi(\hat{x})=z x .
$$

Calculating the individual terms we have

$$
\begin{aligned}
\frac{\partial \psi(\hat{x})}{\partial \hat{x}}\left(\begin{array}{l}
u \\
v
\end{array}\right) & =\lim _{h \rightarrow 0} \frac{(x+h u)(z+h v)-z x}{h} \\
& =z u+v x
\end{aligned}
$$


and so we have

$$
\left\langle\frac{\partial \psi}{\partial \hat{x}}, f(\hat{x})\right\rangle=z((z A+(1-z) B) x=A z x
$$

since $z^{2}=z$. Thus,

$$
\psi(\hat{x}+g(\hat{x}))-\psi(\hat{x})=x-z x .
$$

Finally, we have that

$$
d(z x)=A z x d t+(x-2 z x) d N
$$

and from this we can calculate the expected values

$$
\frac{d}{d t} E(z x)(t)=A E(z x)(t)+(E(x)-2 E(z x)) \lambda .
$$

Combining equations (2.2) and (2.6) we have the linear system

$$
\frac{d}{d t}\left(\begin{array}{c}
E(x) \\
E(z x)
\end{array}\right)=\left(\begin{array}{cc}
B & A-B \\
\lambda I & A-2 \lambda I
\end{array}\right)\left(\begin{array}{c}
E(x) \\
E(z x)
\end{array}\right) .
$$

To analyze this system, is it is best to make a change of variable

$$
\left(\begin{array}{c}
E(x) \\
E(z x)
\end{array}\right)=\left(\begin{array}{cc}
I & I \\
0 & I
\end{array}\right)\left(\begin{array}{l}
u \\
v
\end{array}\right)
$$

and, after routine manipulations, we have

$$
\frac{d}{d t}\left(\begin{array}{l}
u \\
v
\end{array}\right)=\left(\begin{array}{cc}
B-\lambda I & \lambda I \\
\lambda I & A-\lambda I
\end{array}\right)\left(\begin{array}{l}
u \\
v
\end{array}\right) .
$$

We have proved the following theorem.

THeOREM 2.1. Consider the system

$$
\begin{aligned}
& d x=(z A+(1-z) B) x d t \\
& d z=(1-2 z) d N
\end{aligned}
$$

where $N$ is a Poisson process with rate $\lambda$. The expected value of $x, E x(t)$ goes to zero for all $\lambda$ if and only if the matrix

$$
\left(\begin{array}{cc}
B-\lambda I & \lambda I \\
\lambda I & A-\lambda I
\end{array}\right)
$$

has all of its eigenvalues in the open left half plane.

Corollary 2.2. The expected value of $x(t), E(x(t))$ goes to zero if and only if there is a family of positive definite symmetric matrices

$$
\left(\begin{array}{ll}
P_{1}(\lambda) & P_{2}(\lambda) \\
P_{2}(\lambda) & P_{3}(\lambda)
\end{array}\right)
$$


such that

$$
\begin{gathered}
\left(\begin{array}{cc}
B^{\prime}-\lambda I & \lambda I \\
\lambda I & A^{\prime}-\lambda I
\end{array}\right)\left(\begin{array}{cc}
P_{1}(\lambda) & P_{2}(\lambda) \\
P_{2}(\lambda) & P_{3}(\lambda)
\end{array}\right)+ \\
\left(\begin{array}{cc}
P_{1}(\lambda) & P_{2}(\lambda) \\
P_{2}(\lambda) & P_{3}(\lambda)
\end{array}\right)\left(\begin{array}{cc}
B-\lambda I & \lambda I \\
\lambda I & A-\lambda I
\end{array}\right)=\left(\begin{array}{cc}
-I & 0 \\
0 & -I
\end{array}\right) .
\end{gathered}
$$

3. Analysis of the Expected Value. We analyze the spectrum of the matrix

$$
\mathbf{M}_{0}(\lambda) \equiv\left(\begin{array}{cc}
B-\lambda I & \lambda I \\
\lambda I & A-\lambda I
\end{array}\right) .
$$

At $\lambda=0$ the matrix is block diagonal and is stable if and only if both $A$ and $B$ are stable. Setting $\lambda=0$ is equivalent to saying that the that there is no switching at all. Thus if we start at $\dot{x}=A x$ we stay with this system with probability 1 . Likewise if we start at $\dot{x}=B x$. Since the eigenvalues lie in the open left half plane for small $\lambda$, the system will remain stable.

$$
\mathbf{M}_{1}(\lambda)=\left(\begin{array}{cc}
\frac{1}{\lambda} B-I & I \\
I & \frac{1}{\lambda} A-I
\end{array}\right) .
$$

Taking the limit yields the following interesting matrix

$$
\lim _{\lambda \rightarrow \infty} \mathbf{M}_{1}(\lambda)=\left(\begin{array}{cc}
-I & I \\
I & -I
\end{array}\right) .
$$

The spectrum of this matrix is the two point set $\{0,-2\}$ with each having multiplicity $n$. The case of large $\lambda$ corresponds to very fast switching between the two systems. Here we have a $\mathrm{n}$ example that cannot occur when all switching sequences have equal probability. Consider the case that $A=0$ (scalar) and $B=-\epsilon$ we than have the matrix

$$
\left(\begin{array}{cc}
-\epsilon-1 & 1 \\
1 & -1
\end{array}\right)
$$

The eigenvalues of this matrix are

$$
\frac{1}{2}\left[-\epsilon-2 \pm \sqrt{\epsilon^{2}+4}\right]
$$

Both of these values lie in the open left half plane for all positive $\epsilon$. Thus for $\lambda$ sufficiently large and $\epsilon$ sufficiently small the expected values of $x$ goes to zero. If the switching is sufficiently fast then the switching is dominated by the stable system. This example can be extended to the matrix

$$
\left(\begin{array}{cc}
a-1 & 1 \\
1 & -b-1
\end{array}\right) \text {. }
$$


We now consider when this system is stable. Here we are assuming that both $a$ and $b$ are positive. After some routine calculations we have that the system is stable if and only

$$
a b>a-b .
$$

In particular, if $b>a$ then this inequality always holds. The interpretation is that with very fast switching the system is stable provided the stable system dominates. These argument naturally lead to the following conjecture.

Conjecture 1. Consider the system

$$
\begin{aligned}
& d x=(z A+(1-z) B) x \\
& d z=(1-2 z) d N
\end{aligned}
$$

where $d N$ is a Poisson process of rate $\lambda$. If $A$ is stable and $\gamma \in \operatorname{Spectrum}(A)$ and $\rho \in \operatorname{Spectrum}(B)$ implies

$$
|\gamma+\bar{\gamma}|>|\rho+\bar{\rho}|
$$

then the expected value of $x$ goes to 0 with time.

Consider the control system

$$
\frac{d}{d t}\left(\begin{array}{l}
x \\
z
\end{array}\right)=\left(\begin{array}{cc}
-I & I \\
I & -I
\end{array}\right)\left(\begin{array}{l}
x \\
z
\end{array}\right)+\left(\begin{array}{ll}
I & 0 \\
0 & I
\end{array}\right)\left(\begin{array}{l}
u \\
v
\end{array}\right)
$$

and now ask when this system can be stabilized using restricted feedback of the form $u=B x$ and $v=A z$. A similar problem is the stabilization of the complex system

$$
\dot{z}=(F+i G) x+H u
$$

using real feedback, $[7,10]$. This problem remains open which would suggest that the above problem may also be quite difficult.

We will continue this analysis in Section 5 .

4. Higher Order Moments. In this section we return to analyzing the switched linear system represented by equation (1.1). Specifically, we proceed by finding an equation for the homogeneous monoids of degree $p$. Let $X_{[p]}$ represent the vector of all monoids of degree $p$, so that

$$
X_{[p]}=\left(\begin{array}{c}
x_{1}^{p} \\
x_{1}^{p-1} x_{2} \\
\vdots \\
x_{n-1} x_{n}^{p-1} \\
x_{n}^{p}
\end{array}\right) .
$$


We will find matrices

$$
A_{[p]} \text { and } B_{[p]}
$$

so that

$$
\frac{d}{d t} X_{[p]}=\left[\delta(t) A_{[p]}+(1-\delta(t)) B_{[p]}\right] X_{[p]} .
$$

To see the existence of these matrices consider

$$
\frac{d}{d t} x_{1}^{p_{1}} x_{2}^{p_{2}} \cdots x_{n}^{p_{n}}=\sum_{i=1}^{n} p_{i} x_{i}^{p_{i}-1} x_{1}^{p_{1}} x_{2}^{p_{2}} \cdots \overline{x_{i}^{p_{i}}} \cdots x_{n}^{p_{n}} \dot{x}_{i}
$$

where the "overline" indicates that term is removed and $p_{1}+\cdots+p_{n}=p$. Let $A_{i}$ indicate the $i^{\text {th }}$ row of the matrix $A$. Now

$$
\dot{x}_{i}=\left[\delta(t) A_{i}+(1-\delta(t)) B_{i}\right] x
$$

and note that this linear in $x$. Thus we get

$$
\begin{aligned}
\frac{d}{d t} x_{1}^{p_{1}} x_{2}^{p_{2}} \cdots x_{n}^{p_{n}}= & \sum_{i=1}^{n} p_{i} x_{i}^{p_{i}-1} x_{1}^{p_{1}} x_{2}^{p_{2}} \cdots \overline{x_{i}^{p_{i}}} \cdots x_{n}^{p_{n}} \dot{x}_{i} \\
= & \left.\sum_{i=1}^{n} p_{i} x_{i}^{p_{i}-1} x_{1}^{p_{1}} x_{2}^{p_{2}} \cdots \overline{x_{i}^{p_{i}}} \cdots x_{n}^{p_{n}}\left[\delta(t) A_{i}+(1-\delta(t)) B_{i}\right)\right] x \\
= & \delta(t)\left(\sum_{i=1}^{n} p_{i} x_{i}^{p_{i}-1} x_{1}^{p_{1}} x_{2}^{p_{2}} \cdots \overline{x_{i}^{p_{i}}} \cdots x_{n}^{p_{n}} A_{i}\right) x+ \\
& (1-\delta(t))\left(\sum_{i=1}^{n} p_{i} x_{i}^{p_{i}-1} x_{1}^{p_{1}} x_{2}^{p_{2}} \cdots \overline{x_{i}^{p_{i}}} \cdots x_{n}^{p_{n}} B_{i}\right) x \\
= & \delta(t) A_{[p], \tau} X_{[p]}+(1-\delta(t)) B_{[p], \tau} X_{[p]}
\end{aligned}
$$

where $\tau$ indicates the row associated with the multi-index $\left(p_{1}, \cdots, p_{n}\right)$. Doing the calculation for each monoid we then have

$$
\frac{d}{d t} X_{[p]}=\left[\delta(t) A_{[p]}+(1-\delta(t)) B_{[p]}\right] X_{[p]} .
$$

Now reformulating this as a stochastic process we have

$$
d\left(\begin{array}{c}
X_{[p]} \\
z
\end{array}\right)=\left(\begin{array}{c}
\left(z A_{[p]}+(1-z) B_{[p]}\right)_{[p]} \\
0
\end{array}\right) d t+\left(\begin{array}{c}
0 \\
1-2 z
\end{array}\right) d N
$$

and calculating expected values as before we have

$$
\frac{d}{d t}\left(\begin{array}{c}
E\left(X_{[p]}\right) \\
E\left(z X_{[p]}\right)
\end{array}\right)=\left(\begin{array}{cc}
B_{[p]} & A_{[p]}-B_{[p]} \\
\lambda I & A_{[p]}-2 \lambda I
\end{array}\right)\left(\begin{array}{c}
E\left(X_{[p]}\right) \\
E\left(z X_{[p]}\right)
\end{array}\right) .
$$


To solve this system is it is best to make a change of variable

$$
\left(\begin{array}{c}
E\left(X_{[p]}\right) \\
E\left(z X_{[p]}\right)
\end{array}\right)=\left(\begin{array}{cc}
I & I \\
0 & I
\end{array}\right)\left(\begin{array}{c}
u \\
v
\end{array}\right)
$$

and after some routine manipulation we have

$$
\frac{d}{d t}\left(\begin{array}{c}
u \\
v
\end{array}\right)=\left(\begin{array}{cc}
B_{[p]}-\lambda I & \lambda I \\
\lambda I & A_{[p]}-\lambda I
\end{array}\right)\left(\begin{array}{l}
u \\
v
\end{array}\right) .
$$

Theorem 4.1. Consider the system

$$
\begin{aligned}
d X_{[p]} & =\left(z A_{[P]}+(1-z) B_{[p]}\right) X_{[p]} d t \\
d z & =(1-2 z) d N
\end{aligned}
$$

where $N$ is a Poisson process with rate $\lambda$. The expected value of $X_{[p]}, E X_{[p]}(t)$ goes to zero for all $\lambda$ if and only if the matrix

$$
\left(\begin{array}{cc}
B_{[p]}-\lambda I & \lambda I \\
\lambda I & A_{[P]}-\lambda I
\end{array}\right)
$$

has all of its eigenvalues in the open left half plane.

Corollary 4.2. The expected value of $x(t), E(x(t))$ goes to zero if and only if there is a family of positive definite symmetric matrices

$$
\left(\begin{array}{ll}
P_{1}(\lambda) & P_{2}(\lambda) \\
P_{2}(\lambda) & P_{3}(\lambda)
\end{array}\right)
$$

such that

$$
\begin{gathered}
\left(\begin{array}{cc}
B_{[p]}^{\prime}-\lambda I & \lambda I \\
\lambda I & A_{[P]}^{\prime}-\lambda I
\end{array}\right)\left(\begin{array}{cc}
P_{1}(\lambda) & P_{2}(\lambda) \\
P_{2}(\lambda) & P_{3}(\lambda)
\end{array}\right)+ \\
\left(\begin{array}{cc}
P_{1}(\lambda) & P_{2}(\lambda) \\
P_{2}(\lambda) & P_{3}(\lambda)
\end{array}\right)\left(\begin{array}{cc}
B_{[p]}-\lambda I & \lambda I \\
\lambda I & A_{[P]}-\lambda I
\end{array}\right)=\left(\begin{array}{cc}
-I & 0 \\
0 & -I
\end{array}\right) .
\end{gathered}
$$

It is tempting to conjecture that the system 1.1 is stable for all possible switches if and only if there exists a doubly indexed family of positive definite matrices, $Q_{p, \lambda}$,

$$
\left(\begin{array}{cc}
Q_{1, p, \lambda}(\lambda) & Q_{2, p, \lambda}(\lambda) \\
Q_{2, p, \lambda}(\lambda) & Q_{3, p, \lambda}(\lambda)
\end{array}\right)
$$

such that

$$
\begin{gathered}
\left(\begin{array}{cc}
B_{[p]}^{\prime}-\lambda I & \lambda I \\
\lambda I & A_{[P]}^{\prime}-\lambda I
\end{array}\right)\left(\begin{array}{cc}
Q_{1, p, \lambda}(\lambda) & Q_{2, p, \lambda}(\lambda) \\
Q_{2, p, \lambda}(\lambda) & Q_{3, p, \lambda}(\lambda)
\end{array}\right)+ \\
\left(\begin{array}{cc}
Q_{1, p, \lambda}(\lambda) & Q_{2, p, \lambda}(\lambda) \\
Q_{2, p, \lambda}(\lambda) & Q_{3, p, \lambda}(\lambda)
\end{array}\right)\left(\begin{array}{cc}
B_{[p]}-\lambda I & \lambda I \\
\lambda I & A_{[P]}-\lambda I
\end{array}\right)=\left(\begin{array}{cc}
-I & 0 \\
0 & -I
\end{array}\right) .
\end{gathered}
$$

We will later prove that if all of the solutions of equation (1.1) are exponentially stable then the all of the expectations are likewise exponentially stable. However, the converse is false as we will see in the next section. 
5. A fundamental Example. In [5] the following example was constructed.

$$
\begin{gathered}
\frac{d}{d t}\left(\begin{array}{l}
x_{1} \\
x_{2}
\end{array}\right)=\left[\delta(t)\left(\begin{array}{cc}
-1 & -1 \\
1 & -1
\end{array}\right)+(1-\delta(t))\left(\begin{array}{cc}
-1 & -a \\
1 / a & -1
\end{array}\right)\right]\left(\begin{array}{l}
x_{1} \\
x_{2}
\end{array}\right)= \\
(\delta(t) A+(1-\delta(t)) B) x
\end{gathered}
$$

where $a \geq 1$. Note that $A$ and $B$ have the same eigenvalues and that the orbits of $B$ are simply elongated orbits of $A$. In the paper they proved that the set of $a$ 's for which there exists a quadratic Lyapunov function is contained in the interval $[1,6]$ and the set of $a$ 's for which the system is exponentially stable contains the interval $[1,10]$. Thus, in the interval $(6,10)$ there exists a Lyapunov function but there does not exist a quadratic Lyapunov function. We will examine this example in more detail in this section.

We begin by determining an upper bound for the $a$ 's for which the system is stable for all switches. First we note that

$$
e^{A t}=\left(\begin{array}{cc}
e^{-t} \cos t & e^{-t} \sin t \\
-e^{-t} \sin t & e^{-t} \cos t
\end{array}\right) \text { and } e^{B t}=\left(\begin{array}{cc}
e^{-t} \cos t & a e^{-t} \sin t \\
\frac{-e^{-t} \sin t}{a} & e^{-t} \cos t
\end{array}\right) .
$$

Let the initial data be $(0,1)^{t}$ and start with $e^{B t}$ and then switch at $t=\pi / 2$ to be at

$$
\left(\begin{array}{c}
a e^{-\pi / 2} \\
0
\end{array}\right)
$$

Now we drive the system with $e^{A t}$ to move along the curve

$$
\left(\begin{array}{c}
a e^{-\pi / 2} e^{-t} \cos t \\
a e^{-\pi / 2} e^{-t} \sin t
\end{array}\right)
$$

for $\pi / 2$ units to arrive at

$$
\left(\begin{array}{c}
0 \\
a e^{-\pi}
\end{array}\right)
$$

In this example, the system started at started at $(0,1)^{t}$; if it is going to diverge to infinity, then we must have that $a e^{-\pi}>1$. Letting $a=25$ suffices. So, we have shown that if $a \in[25, \infty)$ then the system is unstable.

We now consider the matrix coming from the expectation

$$
H=\left(\begin{array}{cccc}
-1-\lambda & -1 & \lambda & 0 \\
1 & -1-\lambda & 0 & \lambda \\
\lambda & 0 & -1-\lambda & -a \\
0 & \lambda & 1 / a & -1-\lambda
\end{array}\right)
$$


Here our goal is to find a lower bound on the $a$ 's such that there is a $\lambda$ for which the matrix is unstable. The eigenvalues of $H$ are

$$
-1-\lambda \pm \sqrt{-1+\lambda^{2} \pm \lambda \sqrt{-2-a-1 / a}} .
$$

The only two of these numbers that can be positive are

$$
-1-\lambda+\sqrt{-1+\lambda^{2} \pm \lambda \sqrt{-2-a-1 / a}} .
$$

We calculate

$$
\sqrt{-1+\lambda^{2}+\lambda \sqrt{-2-a-1 / a}}=-1+\lambda^{2}+i \lambda c
$$

where $c=\sqrt{2+a+1 / a}$. Now, the real part of $1-\lambda+\sqrt{-1+\lambda^{2} \pm \lambda \sqrt{-2-a-1 / a}}$ is given by

$$
g(\lambda, a)=-1-\lambda+\frac{1}{\sqrt{2}} \sqrt{-1+\lambda^{2}+\sqrt{\left(1-\lambda^{2}\right)^{2}+\lambda^{2} c^{2}}}
$$

Therefore, the question reduces to determining the smallest value of $a$ for which $g(\lambda, a)=0$ for some positive value of $\lambda$. This equation is a difficult to solve because of extraneous roots when it is squared. However, it is easy to show that if $a<52$, then no positive $\lambda$ makes the equation equal to 0 . Figure 1 displays a graph of $g(\lambda, a)$ for $1<\lambda<5$ and $1<a<52$; Figure 2 displays a graph of $g(\lambda, a)$ for $a=52$ and $0<\lambda<5$.

Thus the expected value of $x$ goes to zero for all $a \in[1,52]$. We collect these facts in the following theorem.

THEOREM 5.1. Consider the system

$$
\Sigma_{1}: \frac{d}{d t}\left(\begin{array}{l}
x_{1} \\
x_{2}
\end{array}\right)=\left[\delta(t)\left(\begin{array}{cc}
-1 & -1 \\
1 & -1
\end{array}\right)+(1-\delta(t))\left(\begin{array}{cc}
-1 & -a \\
1 / a & -1
\end{array}\right)\right]\left(\begin{array}{l}
x_{1} \\
x_{2}
\end{array}\right)
$$

and the associated stochastic process

$$
\begin{aligned}
\Sigma_{2}: \quad d\left(\begin{array}{l}
x_{1} \\
x_{2}
\end{array}\right) & =\left[z\left(\begin{array}{cc}
-1 & -1 \\
1 & -1
\end{array}\right)+(1-z)\left(\begin{array}{cc}
-1 & -a \\
1 / a & -1
\end{array}\right)\right]\left(\begin{array}{l}
x_{1} \\
x_{2}
\end{array}\right) d t \\
d z & =(1-2 z) d N .
\end{aligned}
$$

1. There exists a quadratic Lyapunov function for $\Sigma_{1}$ for $a \in[1, \alpha]$ where $\alpha<6$, [5].

2. The system $\Sigma_{1}$ is stable for $a \in[1,10]$ and hence there is a non-quadratic Lyapunov function for the system $\Sigma_{1}$ in this interval, [5].

3. The system $\Sigma_{1}$ is unstable for $a \in[25, \infty)$.

4. For the system $\Sigma_{2}$ the expected value of $x$ goes to zero with time for a $\in[1,52]$ and for all $\lambda$. 


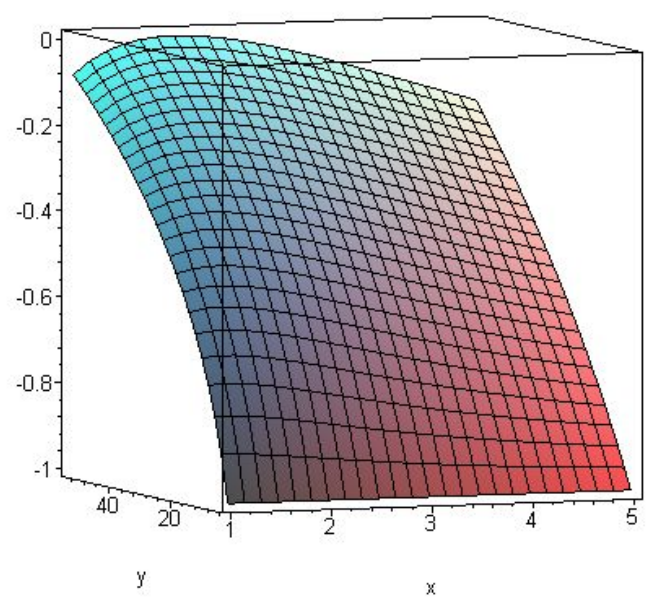

FIG. 1. The graph of real part of the eigenvalue

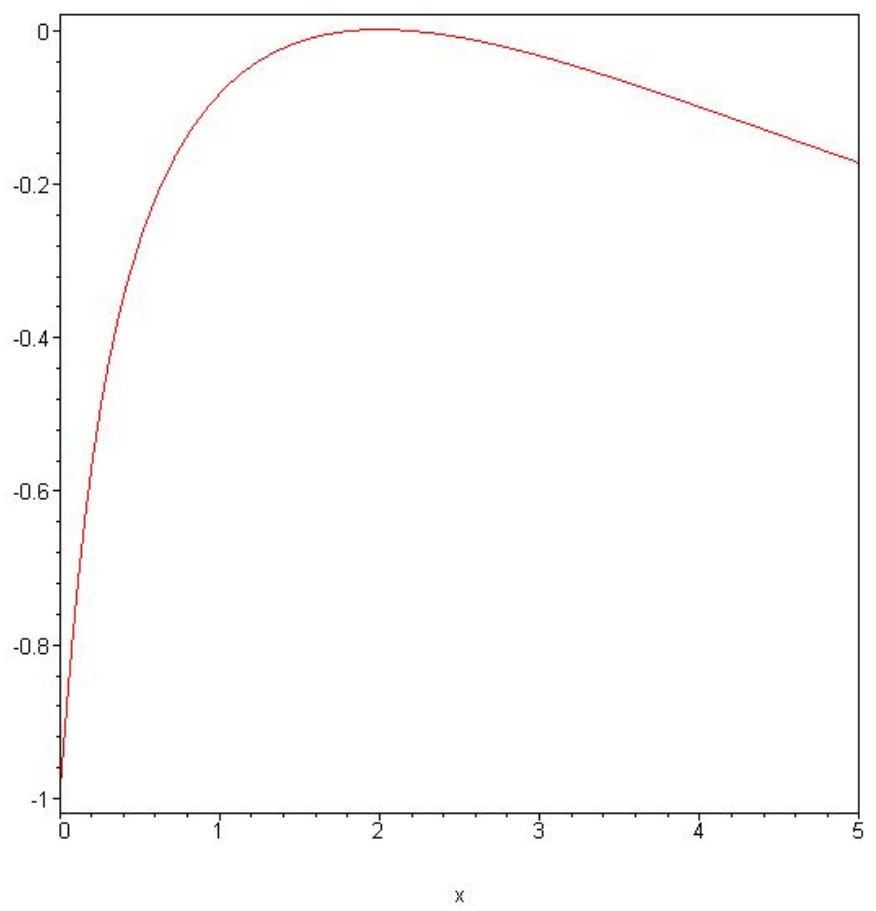

FIG. 2. The graph of $g$ with a chosen so that the $g<0$. 
5.1. Second Moments. We now calculate the second moments for the above system. First we will compute the second moments of a pair of second order linear systems. Let

$$
A=\left(\begin{array}{ll}
a_{11} & a_{12} \\
a_{21} & a_{22}
\end{array}\right) \text { and } B=\left(\begin{array}{ll}
b_{11} & b_{12} \\
b_{21} & b_{22}
\end{array}\right)
$$

Then we have

$$
\begin{aligned}
\frac{d}{d t} x_{1}^{2}= & 2 x_{1} \dot{x}_{1} \\
= & 2 x_{1}\left[\delta(t)\left(a_{11} x_{1}+a_{12} x_{2}\right)+(1-\delta(t))\left(b_{11} x_{1}+b_{12} x_{2}\right)\right] \\
= & {\left[\delta(t)\left(2 a_{11} x_{1}^{2}+2 a_{12} x_{1} x_{2}\right)+(1-\delta(t))\left(2 b_{11} x_{1}^{2}+2 b_{12} x_{1} x_{2}\right)\right.} \\
\frac{d}{d t} x_{1} x_{2}= & x_{2} \dot{x}_{1}+x_{1} \dot{x}_{2} \\
= & {\left[\delta(t)\left(a_{11} x_{1} x_{2}+a_{12} x_{2}^{2}\right)+(1-\delta(t))\left(b_{11} x_{1} x_{2}+b_{12} x_{2}^{2}\right)\right]+} \\
& {\left[\delta(t)\left(a_{21} x_{1}^{2}+a_{22} x_{1} x_{2}\right)+(1-\delta(t))\left(b_{21} x_{1}^{2}+b_{22} x_{1} x_{2}\right)\right] } \\
= & \delta(t)\left(a_{21} x_{1}^{2}+\left(a_{11}+a_{22}\right) x_{1} x_{2}+a_{12} x_{2}^{2}\right)+ \\
& (1-\delta(t))\left(\left(b_{21} x_{1}^{2}+b_{11}+b_{22}\right) x_{1} x_{2}+b_{12} x_{2}^{2}\right)
\end{aligned}
$$

and

$$
\begin{aligned}
\frac{d}{d t} x_{2}^{2} & =2 x_{2} \dot{x}_{2} \\
& =2 x_{2}\left[\delta(t)\left(a_{21} x_{1}+a_{22} x_{2}\right)+(1-\delta(t))\left(b_{21} x_{1}+b_{22} x_{2}\right)\right] \\
& =\left[\delta(t)\left(2 a_{21} x_{1} x_{2}+2 a_{22} x_{2}^{2}\right)+(1-\delta(t))\left(2 b_{21} x_{1} x_{2}+2 b_{22} x_{2}^{2}\right)\right] .
\end{aligned}
$$

We can now write down the system

$$
\begin{aligned}
\frac{d}{d t}\left(\begin{array}{c}
x_{1}^{2} \\
x_{1} x_{2} \\
x_{2}^{2}
\end{array}\right)= & {\left[\delta(t)\left(\begin{array}{ccc}
2 a_{11} & 2 a_{12} & 0 \\
a_{21} & a_{11}+a_{22} & a_{12} \\
0 & 2 a_{21} & 2 a_{22}
\end{array}\right)+\right.} \\
& \left.(1-\delta(t))\left(\begin{array}{ccc}
2 b_{11} & 2 b_{12} & 0 \\
b_{21} & b_{11}+b_{22} & b_{12} \\
0 & 2 b_{21} & 2 b_{22}
\end{array}\right)\right]\left(\begin{array}{c}
x_{1}^{2} \\
x_{1} x_{2} \\
x_{2}^{2}
\end{array}\right) .
\end{aligned}
$$

Now for the example above we have

$$
A_{[2]}=\left(\begin{array}{ccc}
-2 & -2 & 0 \\
1 & -2 & -1 \\
0 & 2 & -2
\end{array}\right) \text { and } B_{[2]}=\left(\begin{array}{ccc}
-2 & -2 a & 0 \\
1 / a & -2 & -a \\
0 & 2 / a & -2
\end{array}\right) .
$$

It is easy to see that the characteristic polynomial of $B_{[2]}$ is independent of $a$ and hence is the same as $A_{[2]}$. 


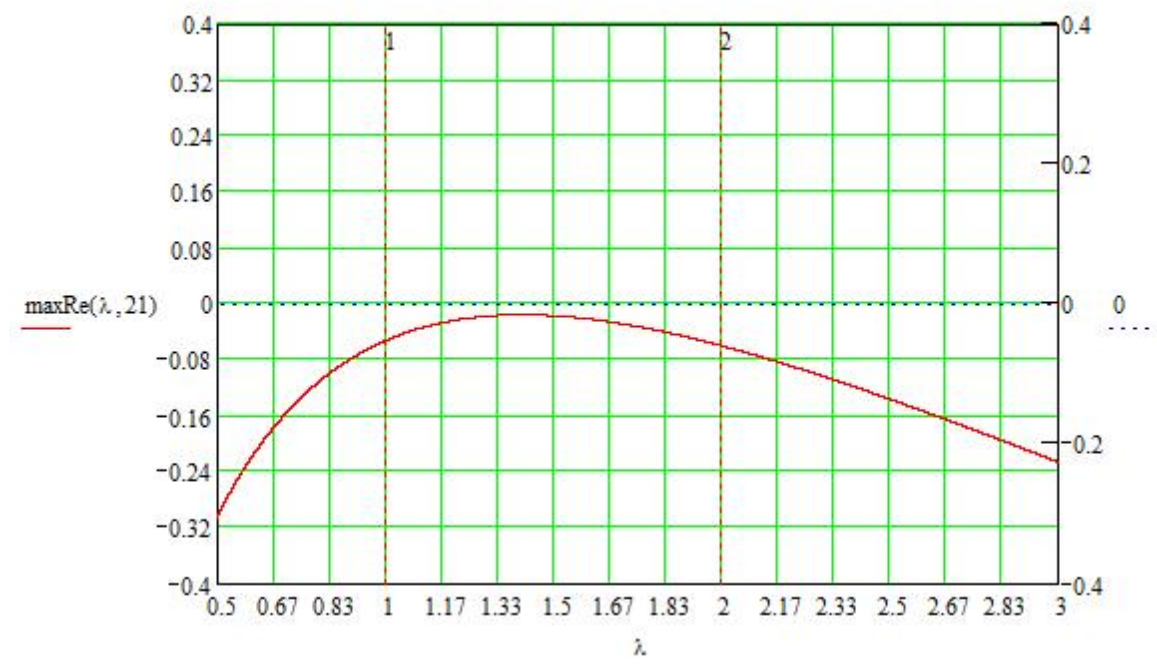

FIG. 3. Showing a lower bound for a.

We are now concerned with the stability of the $6 \times 6$ matrix

$$
H(a, \lambda)=\left(\begin{array}{cccccc}
-2-\lambda & -2 & 0 & \lambda & 0 & 0 \\
1 & -2-\lambda & -1 & 0 & \lambda & 0 \\
0 & 2 & -2-\lambda & 0 & 0 & \lambda \\
\lambda & 0 & 0 & -2-\lambda & -2 a & 0 \\
0 & \lambda & 0 & 1 / a & -2-\lambda & -a \\
0 & 0 & \lambda & 0 & 2 / a & -2-\lambda
\end{array}\right) .
$$

Because the matrix is $6 \times 6$, Maple (or other symbolic packages) will no longer work to find the eigenvalues as explicit functions of $a$ and $\lambda$; we therefore utilize a numerical program to find the values of $a$ for which the matrix is stable for every value of $\lambda$. We define a function

$$
\max R(a, \lambda)=\max \left\{\operatorname{Real}\left(\gamma_{i}(a, \lambda)\right): i=1, \cdots, 6\right\}
$$

where the $\gamma_{i}(a, \lambda)$ are the 6 eigenvalues of $H(a, \lambda)$. We have not attempted to find the exact value of $a$ but from the graphs in Figures 3 and 4 we see that the value is between 21 and 24 . Thus the second moments converge to zero whenever $a \leq 21$. We could continue to calculate the higher moments but we do not expect to see anything very dramatic.

6. Analysis of the Expected value of the Example. The expected value of $x$ is controlled by the eigenvalues

$$
-1-\lambda \pm \sqrt{-1+\lambda^{2} \pm \lambda \sqrt{-2-a-1 / a}}
$$




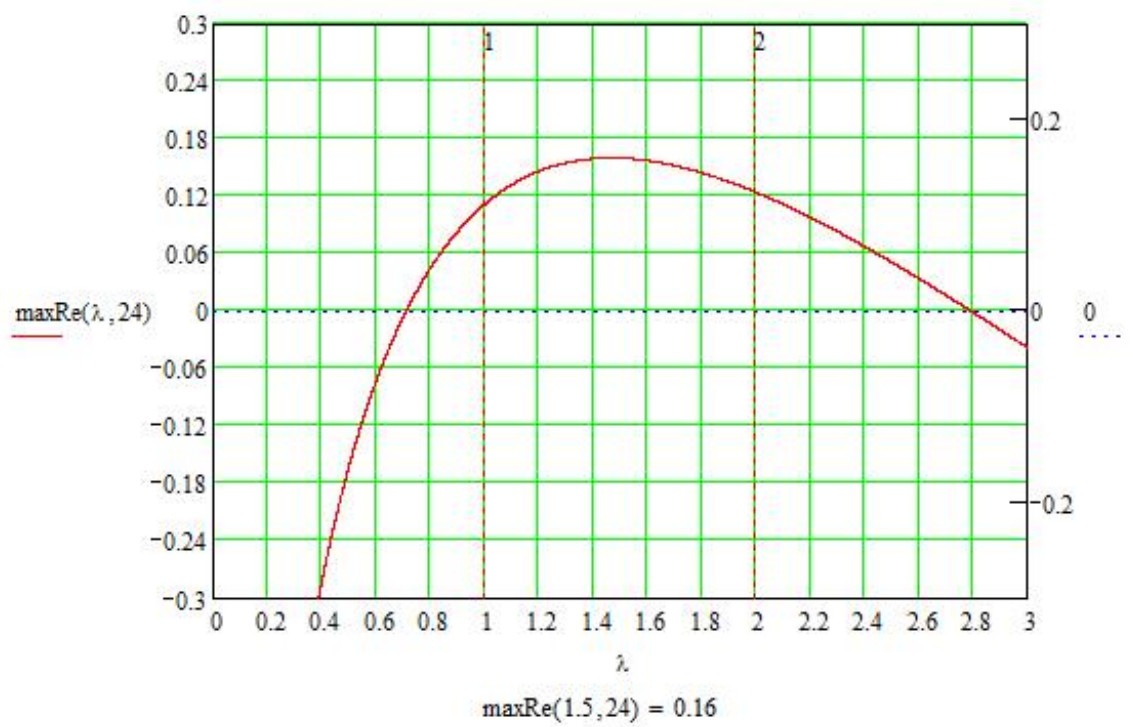

FIG. 4. Showing an upper bound for a.

and the stability is controlled by the real part

$$
g(\lambda, a)=-1-\lambda+\frac{1}{\sqrt{2}} \sqrt{-1+\lambda^{2}+\sqrt{\left(1-\lambda^{2}\right)^{2}+\lambda^{2}(a+1 / a+2)}} .
$$

We have seen that the system is stable for $a \in[1,52]$ but note that for any fixed $\lambda$ the system is unstable for $a$ sufficiently large. However, for any fixed $a$ the system is stable for $\lambda$ sufficiently large. Thus we have the system is stable for

$$
\lambda \in[0, \alpha] \cup[\beta, \infty)
$$

for some value of $\alpha$ and $\beta$. We have seen above that the second moments are stable for $a \in[1,21]$ and as for the expected value that for fixed $a$ there is an $\alpha^{\prime}$ and $\beta^{\prime}$ such that for $\lambda \in\left[0, \alpha^{\prime}\right) \cup\left(\beta^{\prime}, \infty\right)$ the second moments converge to zero. It is beyond the scope of this paper to calculate the entire probability distribution.

7. Stability. In this section we collect some results on the stability of the system. We first note that for the system given by equation (1.1) that if it is stable for all possible switching sequences then all moments converge to zero. We state this a theorem.

Theorem 7.1. Assume that $A$ and $B$ are such that the system

$$
\dot{x}(t)=(\delta(t) A+(1-\delta(t)) b) x(t)
$$

is uniformly asymptotically stable for all possible switching sequences $\delta(t)$. Then for every $p$ and every $\lambda \geq 0$ the expected value,

$$
E X_{[p]} \rightarrow 0
$$


The proof is immediate.

Now we consider the system

$$
\frac{d}{d t}\left(\begin{array}{l}
u \\
v
\end{array}\right)=\left(\begin{array}{cc}
B_{[p]}-\lambda I & \lambda I \\
\lambda I & A_{[p]}-\lambda I
\end{array}\right)\left(\begin{array}{l}
u \\
v
\end{array}\right) .
$$

For $p=1$ the system is stable for $\lambda=0$ and hence is stable for some interval containing 0 . We will now prove that this holds for all $p$. We begin with a technical result.

Theorem 7.2. Let $A$ be an $n \times n$ matrix such that $\gamma \in \operatorname{Spec}(A)$ implies that $\gamma+\bar{\gamma}<0$ and suppose that there exists an invertible matrix $Q$ such that $Q^{-1} A Q$ is diagonal. Then for very positive integer $p, A_{[p]}$ is diagonalizable and its spectrum lies in the open left half plane.

We first prove a critical lemma.

Lemma 7.3. Let $P z=x x \in R^{n}$ and $P$ an invertible matrix mapping $R^{n}$ to $R^{n}$. Let $V_{k}$ be the space of all homogeneous polynomials of degree $k$. Then the matrix $P_{[k]}$ mapping $V_{[k]}$ to $V_{[k]}$ is an invertible matrix.

Proof. We define a mapping via its actions on a basis.

$$
P_{[k]}\left(z_{1}^{p_{1}} \cdots z_{n}^{p_{n}}\right)=\left(P_{1} z\right)^{p_{1}} \cdots\left(P_{n} z\right)^{p_{n}}
$$

where $P_{i}$ is the $i^{t h}$ row of $P$. Now suppose that there exist constants $\gamma_{\left(p_{1}, \cdots, p_{n}\right)}$ such that

$$
\sum_{\left(p_{1}, \cdots, p_{n}\right)} \gamma_{\left(p_{1}, \cdots, p_{n}\right)}\left(P_{1} z\right)^{p_{1}} \cdots\left(P_{n} z\right)^{p_{n}}=0
$$

Now choose a nonzero vectors $z_{0}$ so that

$$
P_{i} z_{0} \neq 0
$$

and for $j \neq i$

$$
P_{j}\left(z_{0}\right)=0 .
$$

Let

$$
r\left(z_{1}, \cdots, z_{n}\right)=\sum_{\left(p_{1}, \cdots, p_{n}\right)} \gamma_{\left(p_{1}, \cdots, p_{n}\right)}\left(z_{1}\right)^{p_{1}} \cdots\left(z_{n}\right)^{p_{n}} .
$$

Now evaluate

$$
P_{[k]}\left(z_{0}\right)=\gamma_{(0, \cdots, 0, p, 0 \cdots, 0)}\left(P_{i} z_{0}\right)^{p}=0
$$

and hence we have for every $i, \gamma_{(0, \cdots, 0, p, 0 \cdots, 0)}=0$. 
Now choose a family of vectors $z_{t, s}$ such that $P_{i} z_{t . s}=t, P_{j} z_{t, s}=s$ and for every $m \neq i$ or $j, P_{m} z_{t, s}=0$. Then evaluating we have

$$
\begin{aligned}
P_{[k]}\left(z_{t, s}\right) & =\sum_{\rho=1}^{k-1} \gamma_{\rho}\left(P_{i} z_{t, s}\right)^{\rho}\left(P_{j} z_{t, s}\right)^{k-\rho} \\
& =\sum_{\rho=1}^{k-1} \gamma_{\rho} t^{\rho} s^{k-\rho} \\
& =0 .
\end{aligned}
$$

Now set $t=1$ and we have a polynomial in $s$. this polynomial is identically zero and hence its coefficients, $\gamma_{\rho}=0$. Thus we have removed all of the terms that involve one or two variables. Assume that we have shown that any term that involves less than $\mathrm{m}$ terms has a zero coefficient. Now choose a vector $z_{0}$ such that $P_{i_{1}} z_{0}=$ $t_{1}, P_{i_{m}} z_{0}=t_{m}$ and if $\tau$ not in $\left\{i_{1}, \cdots, i_{m}\right\}$ then $P_{\tau} z_{0}=0$. Then evaluating $P_{[k]}\left(z_{0}\right)$ we have a polynomial in the variables $t_{1}, \cdots, t_{m}$ that is identically zero and hence the corresponding coefficients are zero. thus we have shown that if

$$
P[k](r(z))=0
$$

then $r(z)=0$ thus the mapping is one to one. But any linear mapping from $R^{N}$ to $R^{N}$ that is one to one is also onto and hence the matrix $P_{[k]}$ is invertible.

Proof of Theorem: Let $Q$ be such that $Q^{-1} A Q=D$ where $D$ is a diagonal matrix with $d_{i i}+\bar{d}_{i i}<0$. Let $V_{[p]}(\hat{x})$ be the vector space of all homogeneous polynomial of degree $\mathrm{p}$ in the variables $x_{1}, \cdots, x_{n}$. Let $\hat{x}=\left(x_{1}, \cdots, x_{n}\right)^{\prime}$ and define a new set of variables by $\hat{z}=Q \hat{x}$. Let $X_{[p]}$ be the vector of all homogeneous monoids of degree p. Then we define a map $Q_{[p]}$ from $V_{[p]}(\hat{x}) \rightarrow V_{[p]}(\hat{z})$ in a natural manner. For the monoid we have

$$
x_{1}^{p_{1}} \cdots x_{n}^{p_{n}} \rightarrow\left(Q_{1} \hat{z}\right)^{p_{1}} \cdots\left(Q_{n} \hat{z}\right)^{p_{n}} .
$$

Now we have proved in the lemma that $\hat{Q}_{[p]}$ is invertible and so we have that

$$
\hat{Q}^{-1} A_{[p]} \hat{Q}_{[p]}=D_{[p]} .
$$

It only remains to show that $D_{[p]}$ is diagonal. But $D_{[p]}$ is constructed by taking the derivative of

$$
x_{1}^{p_{1}} \cdots x_{n}^{p_{n}}
$$


in doing so we have

$$
\begin{aligned}
\frac{d}{d t} x_{1}^{p_{1}} \cdots x_{n}^{p_{n}} & =\sum_{i=1}^{n} p_{i} x_{1}^{p_{1}} \cdots x_{i-1}^{p_{i-1}} x_{i}^{p_{i}-1} x_{i+1}^{p_{i+1}} \cdots x_{n}^{p_{n}} \dot{x}_{i} \\
& =\sum_{i=1}^{n} p_{i} x_{1}^{p_{1}} \cdots x_{i-1}^{p_{i-1}} x_{i}^{p_{i}-1} x_{i+1}^{p_{i+1}} \cdots x_{n}^{p_{n}} d_{i} x_{i} \\
& =\sum_{i=1}^{n} p_{i} d_{i} x_{1}^{p_{1}} \cdots x_{i-1}^{p_{i-1}} x_{i}^{p_{i}} x_{i+1}^{p_{i+1}} \cdots x_{n}^{p_{n}} \\
& =\left(\sum_{i=1}^{n} p_{i} d_{i}\right) x_{1}^{p_{1}} \cdots x_{n}^{p_{n}} .
\end{aligned}
$$

Thus $D_{[p]}$ is diagonal with the entries being $\sum_{i=1}^{n} p_{i} d_{i}$. Now the $p_{i}$ are non-negative integers and the $d_{i}$ all have negative real parts and hence the sum has negative real part. This finishes the proof of the theorem.

Thus we now have the following.

Theorem 7.4. Consider

$$
d\left(\begin{array}{c}
X_{[p]} \\
z
\end{array}\right)=\left(\begin{array}{c}
\left(z A_{[p]}+(1-z) B_{[p]}\right)_{[p]} \\
0
\end{array}\right) d t+\left(\begin{array}{c}
0 \\
1-2 z
\end{array}\right) d N .
$$

For every $p$ the expected values $E X_{[p]} \rightarrow 0$ and $E z X_{[p]} \rightarrow 0$ for

$$
\lambda \in\left[0, \alpha_{p}\right)
$$

for some $\alpha_{p}>0$.

8. Relation to Random Products of Matrices. Let

$$
S_{A}=\left\{e^{A \alpha}: \alpha \in \mathbb{R}^{+}\right\}
$$

and let

$$
S_{B}=\left\{e^{B \beta}: \beta \in \mathbb{R}^{+}\right\} .
$$

Both $S_{A}$ and $S_{B}$ are semigroups. The solutions of the system (1.1) are then of the form

$$
x(t)=\prod_{i=1} X_{i} x(0)
$$

where

$$
X_{i} \in S_{A} \cup S_{B} .
$$

For the associated stochastic process we select an element $X_{i}$ based on a Poisson process on the real numbers $\alpha$ and $\beta$. This process is very much of the flavor of the 
seminal work of Furstenberg and Keston, [8, 9]. The main difference is that they typically assume that the $X_{i}$ come from a single semigroup and here we are assuming that we are sampling from a union of two semigroups. The second major difference is that we are interested in the case that the products converge to zero, where as in the "products of random matrices" literature the goal is either convergence or to determine the limiting values of the eigenvalues.

9. Conclusion. In this paper we have extended the work on continuous switching system to include the case that switching sequence is governed by a probability distribution. The extension was made by converting the problem to stochastic system and analyzing the stochastic system. Particular attention was paid to an example taken from [5]. Brockett's treatment of stochastic systems with a jump process was fundamental to this work. we produced an infinite series of higher order Lyapunov functions that must be satisfied if the system is stable for all choices of the switching sequence. It remains an open problem to determine if this sequence is necessary and sufficient.

\section{REFERENCES}

[1] B. R. BARmish, Necessary and sufficient conditions for quadratic stability of uncertain systems, J. Optim. Theory Appl., 46(1985), pp. 399-408.

[2] M. S. Branicky, Stability of switched and hybrid systems, in Proc. 33rd IEEE Conf. Decision and Contr., 1994, pp. 3498-3503.

[3] R. Brockett, Volterra series and geometric control theory, Automatica-J. IFAC, 12:2(1976), pp. $167-176$.

[4] R. Brockett, Stochastic Control, lecture notes, 2008.

[5] W. P. Dayawansa and C. Martin, A converse Lyapunov theorem for a class of dynamical systems which undergo switching, IEEE Trans. Automat. Control, 44:4(1999), pp. 751-760.

[6] Y. Du, Simulation of the optimal timing of a multiple drug regime, Master's thesis, Texas Tech University, 2009

[7] F. Fagnani and J. Willems, Representations of Symmetric Linear Dynamical Systems, SiCON, 31(1993), pp. 1267-1293.

[8] H. Furstenberg and H. Kesten, Products of random matrices, Ann. Math. Statist, 31(1960), pp. $457-469$.

[9] H. Furstenberg, Noncommuting random products, Trans. Amer. Math. Soc. 108(1963), pp. $377-428$.

[10] M. Hazewinkel And C. Martin, Symmetric linear systems: an application of algebraic systems theory, International Journal of Control, Volume 37, pp. 1371-1384.

[11] F. Jabbax and W. E. Schmittendorf, A noniterative method for the design of linear controllers, IEEE Trans. Automat. Contr., 35(1990), pp. 954-957.

[12] I. N. KAR, A controller design algorithm for a finite family of linear systems, Int. J. Syst. Sci., 27(1996), pp. 887-893.

[13] D. Liberzon, Switching in systems and control. Systems \& Control: Foundations 8 Applications, Birkhuser Boston, Inc., Boston, MA, 2003.

[14] H. Lin And P.J. Antsaklis, Stability and persistent distrubance attenuation properties for networked control systems: switched systems approach, Inter. J. Control, 78(2005), pp. 
$1447-1458$.

[15] H. Lin AND P.J. Antsaklis, Stability and stabilizability of switched linear systems: a survey of recent results, IEEE TAC, 54(2009), pp. 308-322.

[16] C. Lobry, Controllabilite des systemes nonlineaires, Siam J. Contr. Opt., 8(1970), pp. 573-605.

[17] I. G. Malkin, An the question of reversibility of Lyapunovs theorem on asymptotic stability, Prikl. Mat. Meh., 18(1954), pp. 129-138 (in Russian).

[18] A. S. Monse, Supervisory control of families of linear set-point controllers, Part 1: Exact matching, IEEE Trans. Automat. Contr., 41(1996), pp. 1413-1431.

[19] S. A. Murphy, Optimal dynamic treatment regimes, J. R. Stat. Soc. Ser. B Stat. Methodol. 65:2(2003), pp. 331-366.

[20] S. A. Murphy, M.J. van der LaAn, And J.M. Robins, Marginal mean models for dynamic regimes, J. Amer. Statist. Assoc. 96:456(2001), pp. 1410-1423.

[21] S. A. Murphy and D. Bingham, Screeing experiments for Developing Dynamic Treatment Regimes, Journal of the ASA, Vol 104, 391-408

[22] M. Ni And W. Hu, A Riccati equation approach to the design of linear robust controllers, Automatica, 29(1993), pp. 1603-1605.

[23] F. M. Pait And A. S. Morse, A cyclic switching strategy for parameter adaptive control, IEEE Trans. Automat. Contr., 39(1994), pp. 1172-1183.

[24] P. Peleties and R. DeCarlo, Asymptotic stability of m-switched systems using Lyapunov-like functions, in Proc. Amer. Contr. Conf., Boston, MA, June 1991, pp. 1679-1684.

[25] A. Polpitiya, W. Dayawansa, C. Martin, and B. Ghosh, Geometry and control of human eye movements, IEEE Trans. Automat. Control, 52:2(2007), pp. 170-180.

[26] N. WANG, Switched systems as models of dynamic treatment scheduling, Master's thesis, Texas Tech University, 2009.

[27] N. Wang, M. Egerstedt, and C. Martin, Stability of switched linear systems and the convergence of random products, Proceedng of the IEEE CDC2009, Shanghai, China.

[28] M. A. Wicks, P. Peleties, and R. DeCarlo, Construction of piecewise Lyapunov functions for stabilizing switched systems, in Proc. 33rd IEEE Conf. 